\title{
Research on aerodynamic drag of a solid attitude control thruster based on PWM principle
}

\author{
Zhong Yang ${ }^{1, a}$, Hongwei Yan ${ }^{1}$ Junlong Sun ${ }^{1}$ and Wubin $\mathrm{Li}^{1}$ \\ ${ }^{1}$ College of Transportation, Ludong University, 264025 Yantai, China
}

\begin{abstract}
Solid attitude control thrusters based on PWM principle are widely used in kinetic kill vehicle to control its attitude. In this paper, a kind of solid attitude control thruster is proposed and its piston's motion model is established. One of the most important variables which has significant effect on the validity of the model is aerodynamic drag of rocker arm. We did research on the aerodynamic drag by throttle theory, numerical simulation and experiment. The results were consistent and proved to be valid by testing the pressure in the two control chambers. The result is expected to provide reference for the calculation and test of the aerodynamic drag and design of the solid attitude control.
\end{abstract}

\section{Introduction}

The solid attitude control thruster based on PWM (pulse width modulation) principle is widely used in attitude control system of KKV (kinetic kill vehicle). It works under hot gas generated from the gas generator, so it has the advantages of simply structure, good reliability and good security, etc [1-3]. It's installed on the afterbody of KKV to realize three-axis stability which is pitch, yaw and roll [4]. The thruster receives PWM signal and regulates average thrust by controlling the ratio of the two nozzles' switch time [5]. The performance of the thruster reflects the control precision of the thruster [6,7], so the validity of its mathematic model established is important.

In this paper, we introduced a kind of solid attitude control thruster which works under PWM signal. There is a moving part in the thruster which is piston and its reciprocal motion drives the rocker arm to open or close the two nozzles on both sides. During the motion, the aerodynamic drag has a significant impact on the mathematic model of the thruster, so research on the aerodynamic drag of the rocker arm is important and necessary. We studied the aerodynamic drag by throttle theory, numerical simulation and experiment. The method and result are expected to provide reference for the design and research on the solid attitude control thruster.

\section{Mathematic model}

The solid attitude control thruster designed in this paper is shown in Figure 1. The hot gas flows into the thruster from the inlet and a small part goes into the control chamber through the fixed orifices $f_{1}$ and $f_{3}$ as control media. The adjustable orifices $f_{2}$ and $f_{4}$ are enclosed by the electromagnet and the outlet of the control chamber.
As shown in Figure 1, when the adjustable orifice $f_{2}$ is closed and $f_{4}$ is open, the pressure is increased in control chamber 1 and decreased in control chamber 2 . Then the pressure difference is formed and increased gradually. When it's large enough, the piston is driven to move right. The rocker arm is pulled to revolve around its axis and block the Laval nozzle in the left side. Eventually, the hot gas flows out through the right Laval nozzle to produce thrust.

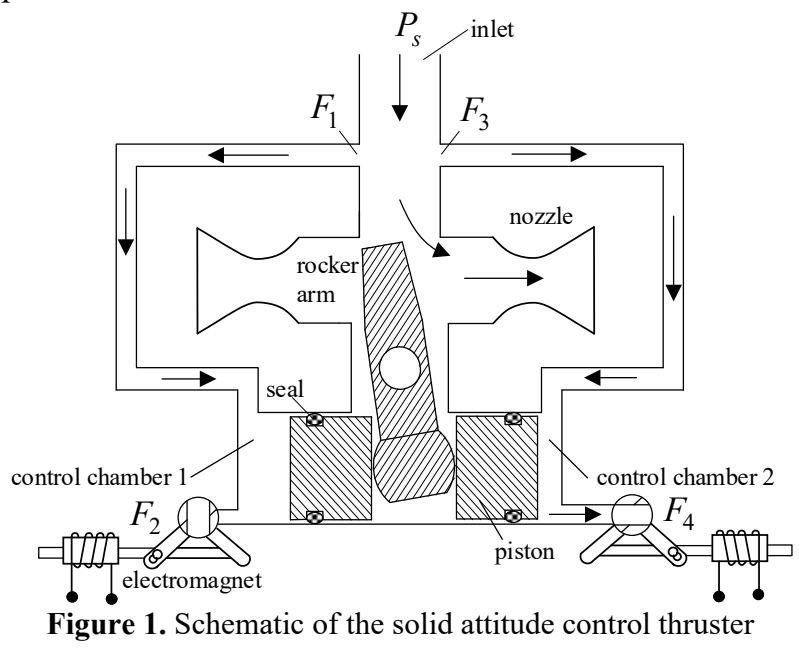

The motion equation of the piston can be written as:

$$
M \ddot{x}=A\left(p_{1}-p_{2}\right)-F_{L}
$$

Where $M, x, A, p_{1}, p_{2}, F_{L}$ mean the mass, displacement, end-face area of the piston, the pressure of the two control chambers and the equivalent drag of the piston respectively.

It's indicated that the equivalent resistance of the piston $F_{L}$ has a significant effect on the piston's motion law from equation (1). The piston needs to overcome frictional drag and aerodynamic drag in motion. The former is generated by the relative motion between the $\mathrm{O}$ ring and the inner surface of thruster's body while the

a Corresponding author: ldu yangzhong@163.com 
latter by the pressure difference between the two sides of the piston during motion. Therefore, the equivalent drag $F_{L}$ is given as follows:

$$
F_{L}=F_{r}+F_{c}
$$

Where $F_{r}$ is the frictional drag and $F_{c}$ is the aerodynamic drag. The frictional drag is related with working pressure, the piston's diameter, the $\mathrm{O}$ ring's material and its decrement, lubricating, etc. The frictional drag is difficult to be obtained by theory, so it was studied by experiment.

The frictional drag is discovered to be much less than the aerodynamic drag which we concentrated on. So we did research on the aerodynamic drag by throttle theory, numerical simulation and experiment.

\section{Analysis of aerodynamic drag}

\subsection{Throttle theory analysis}

The gas flow is complex from the inlet of the thruster to the nozzle by passing through the space between the rocker arm and the body's inner surface. In order to calculate the aerodynamic drag of the rocker arm during motion, we assume that the gas flow status is the same with the steady flow status under the same conditions in every moment. The gas flow through the space between the rocker arm and the body's inner surface into the nozzle can be simplified as through two throttle orifices in series.

The throttle model of the aerodynamic drag is shown in Figure 2. A bridge gas circuit is formed by the four throttle orifices on the two sides. In Figure 2, the two upper throttle orifices $f_{1}$ and $f_{3}$ are the area enclosed by the rocker arm and the body while the lower throttle orifices $f_{2}$ and $f_{4}$ are the nozzle's throat area. The symbol $q_{m a}$ and $q_{m b}$ are the mass flow rate of the gas flowing into or out of the control chambers.

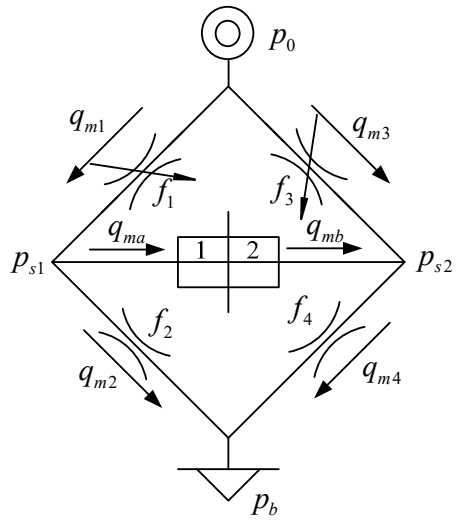

Figure 2. Throttle model of the aerodynamic drag

The pressure between the two throttle orifices $p_{\mathrm{s}}$ is related with the gas source pressure $p_{0}$ and the area ratio of the upper and lower throttle orifices. The pressure $p_{s}$ can be calculated according to the equations shown in Table 1.
Table 1. Equations of calculating the pressure $p_{s}$

\begin{tabular}{|c|c|}
\hline Area ratio & $\boldsymbol{p s}_{\mathbf{s}}$ \\
\hline$C_{t 1}<\frac{f_{1}}{f_{2}}$ & $p_{0}\left[\frac{1}{2}\left(1+\sqrt{1+4\left(\frac{C}{B}\right)^{2}\left(\frac{f_{2}}{f_{1}}\right)^{2}}\right)^{\left(\frac{k}{1-k}\right)}\right.$ \\
\hline$C_{t 1} \leq \frac{f_{1}}{f_{2}} \leq C_{t 2}$ & $p_{0} \frac{f_{1}}{f_{2}}$ \\
\hline $0 \leq \frac{f_{1}}{f_{2}}<C_{t 2}$ & $p_{0} \frac{1}{n}\left[\frac{1}{2}\left(1+\sqrt{1+4 n^{2}\left(\frac{C}{B}\right)^{2}\left(\frac{f_{2}}{f_{1}}\right)^{2}}\right)\right]$ \\
\hline
\end{tabular}

In Table 2, $B$ and $C$ are parameters related with the gas and calculated as follows:

$$
B=\sqrt{\frac{2 k}{R(k-1)}}, C=\left(\frac{2}{1+k}\right)^{\frac{1}{k-1}} \sqrt{\frac{2 k}{R(k+1)}}
$$

In equation (3), $k$ and $R$ are the specific heat ratio and the gas constant of the gas.

The aerodynamic drag can be calculated by converting the pressure difference on the two sides of the rocker arm.

\subsection{Numerical analysis}

The flow field is analysed to calculate the aerodynamic drag by numerical simulation. The control gas circuit has little effect on aerodynamic drag, so computational domain is only set up in flow field of the working chamber around the rocker arm.

The gas's velocity in the inlet of the thruster is low, so the inlet condition and the outlet condition are set as pressure inlet and pressure outlet respectively. The flow field in the thruster is of complex shape and

The grid of the gas flow in the solid attitude control thruster is shown in Figure 3.

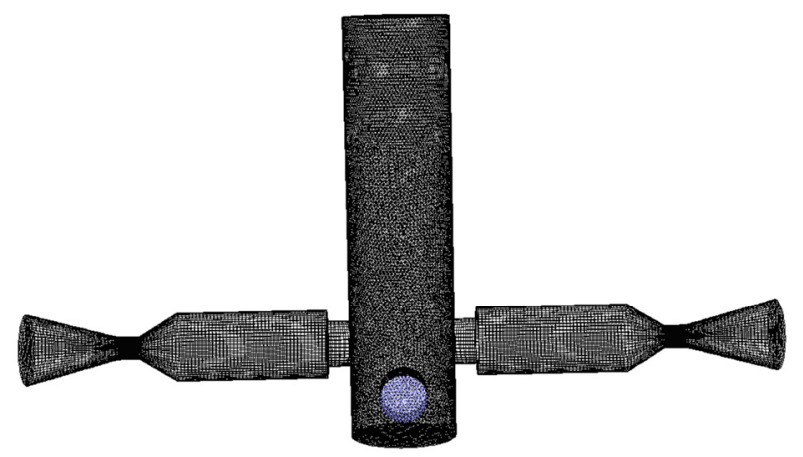

Figure 3. Grid of gas flow in the thruster

Among the turbulent models, Realizable $k-\varepsilon$ model corresponds with the actual model much better and its convergence speed is faster. The simulation results are listed here only when the piston's placement is $0 \mathrm{~mm}$ and $0.8 \mathrm{~mm}$. The pressure nephogram is shown in Figure 4 when the piston's displacement is $0 \mathrm{~mm}$. At this time the pressure on the two sides of the rocker arm is symmetric. The pressure nephogram is shown in Figure 5 and 6 when the piston's displacement is $0.8 \mathrm{~mm}$. Thereinto, Figure 5 shows the pressure nephogram of the closed 
side of the rocker arm. It can be seen that a low pressure appeared in the middle position. Figure 6 shows the pressure nephogram of the open side of the rocker arm and the pressure is almost the same with the gas source.

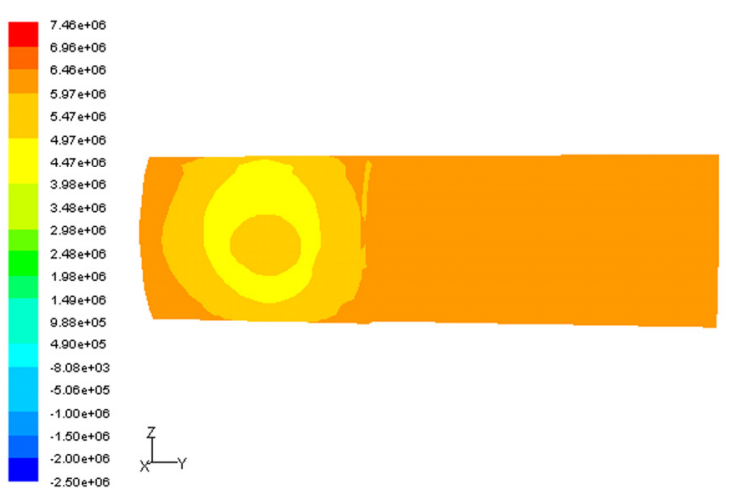

Figure 4. Pressure nephogram when the piston's displacement is $0 \mathrm{~mm}$

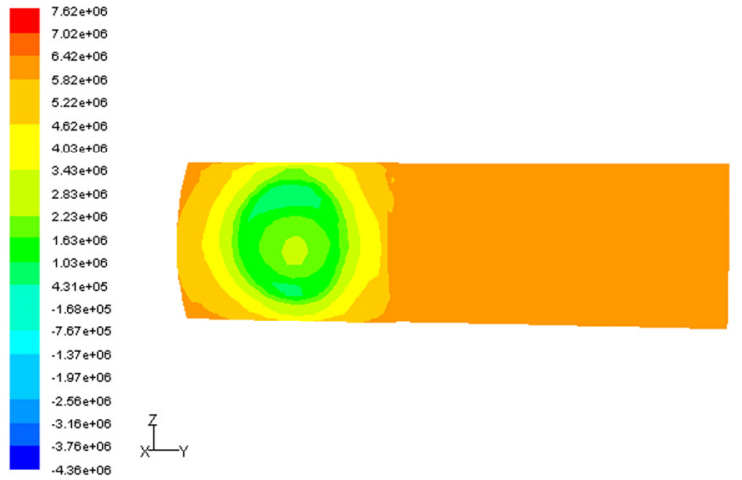

Figure 5. Pressure nephogram of the closed side when the piston's displacement is $0.8 \mathrm{~mm}$

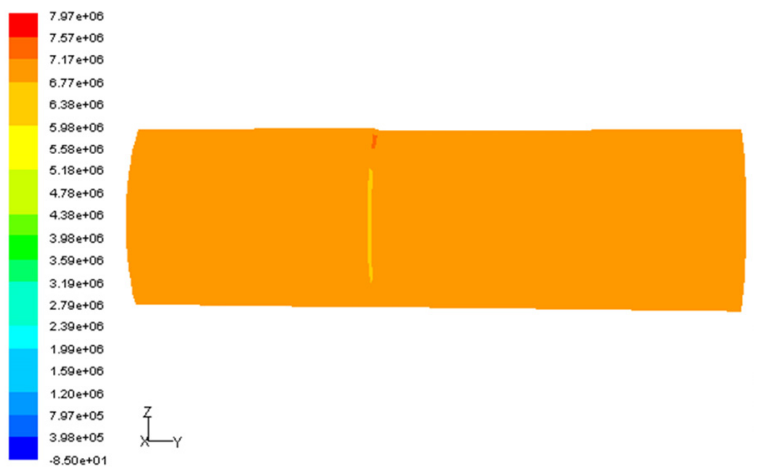

Figure 6. Pressure nephogram of the open side when the piston's displacement is $0.8 \mathrm{~mm}$

\subsection{Experimental analysis}

We did experiments on the aerodynamic drag finally. The experiment principle is shown in Figure 7. We blocked the input of the control chamber so that no gas can go into the control chamber. Then the force sensor and displacement sensor were connected with the piston. During the experiment, the position of the piston was tested by the displacement sensor and the aerodynamic drag was tested by the force sensor. The experiment device is shown in Figure 8.

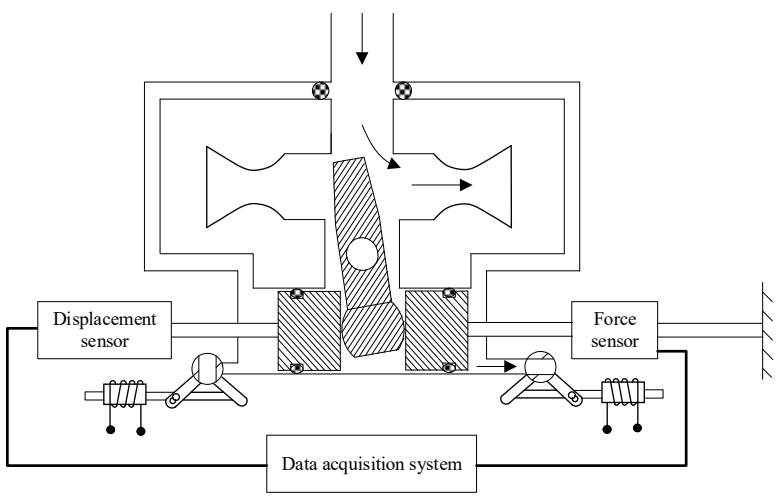

Figure 7. Experiment principle of testing aerodynamic drag

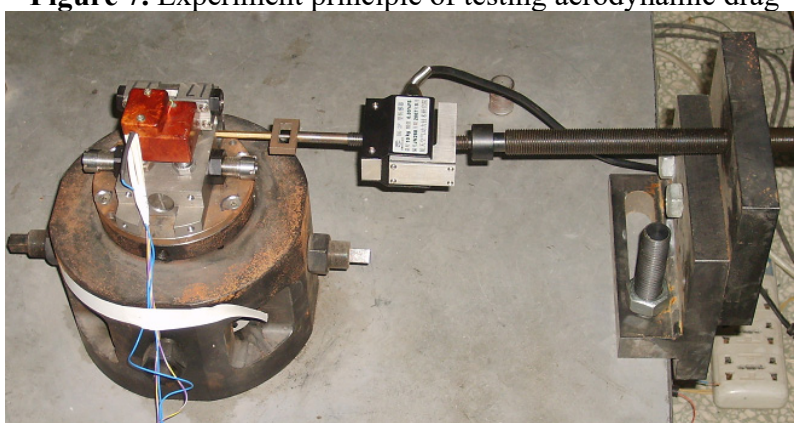

Figure 8. Experiment device of testing aerodynamic drag

\section{Result}

The results of the aerodynamic drag obtained by throttle theory analysis, numerical simulation and experiment are listed in Table 2 and shown in Figure 9.

Table 2. Results data of aerodynamic drag

\begin{tabular}{|c|c|c|c|}
\hline $\begin{array}{c}\text { Piston's } \\
\text { displacement }\end{array}$ & $\begin{array}{c}\text { Throttle } \\
\text { theory }\end{array}$ & $\begin{array}{c}\text { Numerical } \\
\text { simulation }\end{array}$ & $\begin{array}{c}\text { Experimen } \\
\mathbf{t}\end{array}$ \\
\hline-0.8 & -236 & -272.6 & -286.4 \\
\hline-0.6 & -118.4 & -131.8 & -136.5 \\
\hline-0.4 & -58.9 & -59.1 & -60.2 \\
\hline-0.2 & -21.3 & -21.7 & -23.1 \\
\hline 0 & 0 & 0 & 0.3 \\
\hline 0.2 & 21.3 & 21.7 & 22.8 \\
\hline 0.4 & 58.9 & 59.1 & 60.7 \\
\hline 0.6 & 118.4 & 131.8 & 137.3 \\
\hline 0.8 & 236 & 272.6 & 287.1 \\
\hline
\end{tabular}




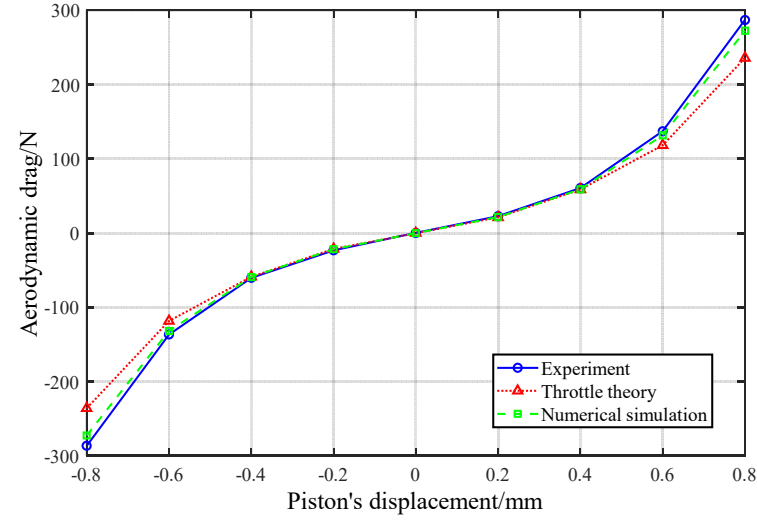

Figure 9. The data of experiment, throttle theory and numerical simulation

It's shown that the changes of all the three groups of data have the same trend and the results had a good symmetry. Meanwhile, the aerodynamic drag changed with the piston's displacement linearly within a small range of the displacement and had an obvious nonlinearity when the displacement increased. This is caused by the nonlinear variation of the flow area between the rocker arm and the body during the rocker arm's motion. Meanwhile, the throttle theory has a high calculation precision within a small range of piston's displacement and the precision is reduced gradually when the displacement increases. That's because the aerodynamic drag is mainly caused by the pressure difference between the two sides of the rocker arm and the throttle theory reflects the changes of the pressure on the two sides. The result of numerical simulation is more consistent with the experiment while it's more complex than throttle theory.

Finally, the piston's motion model is calculated using the experiment data in Table 2. The pressures in the two control chambers are tested and compared with the calculated result to verify the established model's validity. The result is shown in Figure 10.

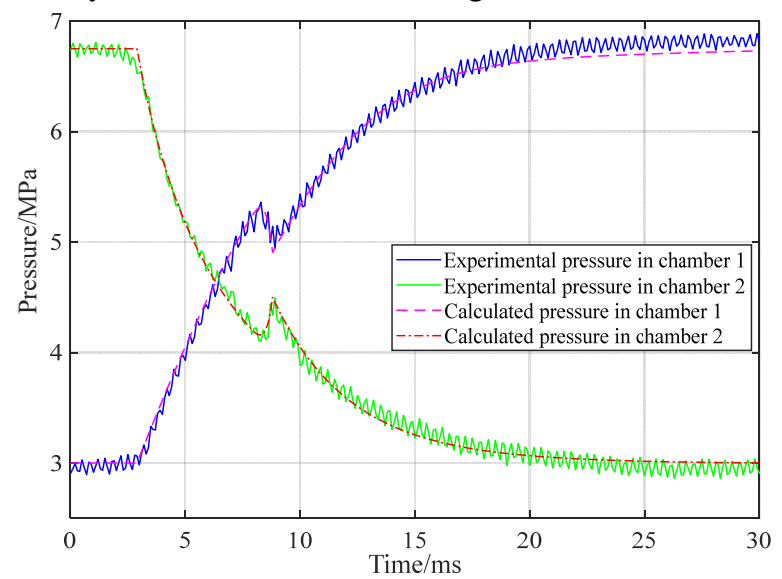

Figure 10. The pressure in the two control chambers

It's demonstrated that the experimental and calculated results were consistent which indicated that the experimental result of the aerodynamic drag was credible. Compared with the experimental result, the throttle theory can be used to calculate preliminarily and the numerical simulation can be used to do further analysis. The result is expected to provide reference for the design of the solid attitude control model.

\section{Conclusion}

In this paper, we proposed a kind of solid attitude control thruster based on PWM principle and the piston's motion model was established. The aerodynamic drag of the rocker arm had significant effect on the validity of the model, so we did research on the aerodynamic drag by throttle theory, numerical simulation and experiment. The results are consistent and can be used to calculate the established model. The results are proved to be valid by testing the pressure in the two control chambers. The method and the results are expected to provide references for the design and analysis of the solid attitude control thrusters.

\section{Acknowledgments}

This work was supported by the Training Fund of Shandong Natural Science Foundation (Grant NO. ZR2017PEE016), the Training Fund of Shandong Natural Science Foundation (Grant NO. ZR2017PEE025) and the Talent Introduction Project of Ludong University (Grant NO. LB2016002).

\section{References}

1. Shipeng Li, Ping Zhang, Analysis of solid propellant control thruster schemes for small KKV. J. Propul. Technol., 20 (1999)

2. Heng Chang, Yibai Wang, Yu Liu, Experimental investigation on solid attitude control system based on PWM principle, J. Solid Rocket Technol., 36 (2013)

3. Xiao Hou, Peng Fu, Yuan Wu, Energy management technology of SRM and its development J. Solid Rocket Technol., 40 (2017)

4. H. Keller, R. Isermann, Model-based nonlinear adaptive control of a pneumatic actuator, Control Eng. Pract., 3 (1993)

5. S. Joner, I. Quinquis, control of an exoatmospheric kill vehicle with a solid propulsion attitude control system, AIAA Guid. Nav. Control Conf. and Exhibit, (2006)

6. Meijun Duan, Di Zhou, Guidance law with finite time convergence considering thrust constraint and dynamics characteristic, J. Syst. Eng. Electron., 39 (2017)

7. Qinghong Liu, Deyuan Chen, Zicai Wang, Effect of system delay on guidance accuracy of interceptor, J. Astronaut 24 (2003) 